\author{
I. Pascher \\ M. Lundmark \\ S. Sundell \\ H. Eibl
}

\section{Conformation and packing of membrane lipids: Crystal structures of lysophosphatidylcholines}

pairs of centrosymmetric $\mathrm{D} / \mathrm{L}$ conformers at either side of the bilayer. In PPC and HPC the $\mathrm{C}_{16}$ hydrocarbon chains interdigitate with an overlap of 13 carbon atoms only, leaving a cavity to accommodate the solvate molecules. The chain matrices have identical tilt $\left(45^{\circ}\right)$, but different chain packing modes $\left(\mathrm{O}^{\prime} \perp\right.$ and $\left.\mathrm{O} \|\right)$. In $\mathrm{PPEM}_{2}$ the chain ends penetrate with 18 -atoms interdigitation to the glycerol region of oppositely oriented molecules and pack in a hybrid matrix with 37 tilt. Despite the differences in hydrocarbon chain attachment (ester/ether) and degree of $N$-methylation the structures show great similarities, in particular with respect to the head group conformation, which apparently is favored by intrinsic energetics.

Key words Phospholipids lysophosphatidylcholines crystal structure - conformation polar interactions

\section{Introduction}

Lysophosphatidylcholines constitute only a minor fraction of the lipid types occurring in biomembranes [1], primarily as intermediates in the turnover of phosphatidylcholines. However, different lysophosphatidylcholine species have been shown to be involved in a variety of biologically important activities $[2,3]$. In particular the ether analogues, alkyl-2-acetyl-glycero-phosphocholines and alkyl-2-methyl-glycero-phosphocholines or related alkyl-phosphocholines were found to function as platelet activating factor (PAF) $[4,5]$ and anti-cancer drugs $[3,6-8]$. respectively. In aqueous dispersions lysophosphatidylcholines exhibit a complex structural behavior forming lamellar, micellar, cubic and hexagonal $\left(\mathrm{H}_{\mathrm{I}}\right)$ phases $[8,9]$. Below the chain melting temperature a lamellar $L_{b i}$ phase with very thin layer thickness is 
observed, indicating that a bilayer structure with interdigitating hydrocarbon chains is formed $[10,11]$.

We have earlier reported on the crystal structure of different glycerophosphocholine compounds such as $D$-glycero-1-phosphocholine ${ }^{1)}$ [12], 3-lauroyl-2-deoxy-glycero1-phosphocholine [13], 3-octadecyl-2-methyl-D-glycero1-phosphocholine [14] and 2,3-dimyristoyl-D-glycero-3phosphocholine [15]. In all these compounds the phosphocholine head groups show very similar conformational features. The packing pattern of the phosphocholine dipoles, however, display great variations.

In the present paper we report on the crystal structure of 3-palmitoyl-D-glycero-1-phosphocholine (PPC), 3-hexadecyl-D-glycero-1-phosphocholine (HPC) and 3-palmitoyl$D L$-glycero-1-phospho- $N, N$-dimethylethanolamine $\left(\mathrm{PPEM}_{2}\right)$. These structure determinations were made to provide further information with atomic resolution on the structure of phosphatidylcholines, in particular on packing features of ester and ether derivatives and on effects on head group conformation and interactions arising from changes in the degree of $\mathrm{N}$-methylation at the ethanolamine nitrogen. With respect to head group interactions lyso-compounds are advantageous as their head group arrangement is usually more relaxed and less affected by chain packing requirements than in corresponding double-chain lipids.

\section{Experimental}

3-Palmitoyl-D-glycero-1-phosphocholine (= 1-palmitoylsn-glycero-3-phosphocholine) (PPC), 3-Hexadecyl-D-glycero-1-phosphocholine ( = 1-hexadecyl-sn-glycero-3-phosphocholine) (HPC) and 3-palmitoyl-DL-glycero-1-phospho$N, N$-dimethylethanolamine ( = 1-palmitoyl-rac-glycero-3phospho- $N, N$-dimethylethanolamine) $\left(\mathrm{PPEM}_{2}\right)$ were sythesized according to Eibl and Woolly [16]. In all compounds homologfree fatty acid or fatty alcohol constituents and reagents of highest purity were used.

Before crystallization the lipids were dried thoroughly in vacuum over $\mathrm{P}_{2} \mathrm{O}_{5}$. Crystals suitable for X-ray single crystal analysis were grown from 1-2\% lipid solutions in anhydrous solvents at $18^{\circ} \mathrm{C}$. For solvent and crystal data see Table 1 . The obtained crystal phases of PPC and HPC,

Table 1

\begin{tabular}{|c|c|c|c|}
\hline Crystal data & $\mathrm{PPC}$ & $\mathrm{HPC}$ & $\mathrm{PPEM}_{2}$ \\
\hline Molecular formula & $\mathrm{C}_{24} \mathrm{H}_{50} \mathrm{NO}_{7} \mathrm{P} \cdot \mathrm{H}_{2} \mathrm{O}$ & $\mathrm{C}_{24} \mathrm{H}_{52} \mathrm{NO}_{6} \mathrm{P} \cdot \mathrm{CHCl}_{3}$ & $\mathrm{C}_{23} \mathrm{H}_{48} \mathrm{NO}_{7} \mathrm{P}$ \\
\hline Molecular weight & 513.65 & 601.03 & 481.61 \\
\hline Crystallization solvent & Ether: ethanol $5: 2^{*}$ & Ether: chloroform $5: 3^{*}$ & Ether: ethanol $5: 2.5^{*}$ \\
\hline Crystal dimensions (mm) & $0.02 \times 0.36 \times 0.48$ & $0.05 \times 0.10 \times 0.29$ & $0.02 \times 0.24 \times 0.43$ \\
\hline recorded $\theta$-range $\left({ }^{\circ}\right)$ & $1<\theta<65$ & $1<\theta<50$ & $1<\theta<45$ \\
\hline Unique/observed reflexions $(I>3 \sigma[I])$ & $2921 / 1004$ & $3351 / 1159$ & $2238 / 1243$ \\
\hline Absorption coefficient $\mu_{\mathrm{CuK} x}\left(\mathrm{~cm}^{-1}\right)$ & 10.87 & 32.70 & 11.90 \\
\hline R-values $R / R_{\mathrm{w}}$ & $0.106 / 0.149$ & $0.127 / 0.164$ & $0.106 / 0.146$ \\
\hline Crystal class & Triclinic & Triclinic & Monoclinic \\
\hline Space group & P1 & P1 & $\mathrm{P} 2_{1} / \mathrm{a}$ \\
\hline Molecules/unit cell $Z$ (independent) & $2(2)$ & $2(2)$ & $4(1)$ \\
\hline \multicolumn{4}{|l|}{ Unit cell parameters (with std's): } \\
\hline$a, b, c(\AA)$ & $5.824(3), 9.857(4), 28.877(15)$ & $5.914(4), 9.864(3), 30.813(7)$ & $11.238(3), 8.586(1), 28.967(6)$ \\
\hline$\alpha, \beta, \gamma()$ & $91.89(4), 90.34(4), 105.67(4)$ & $98.84(2), 102.02(4), 106.95(4)$ & $90.00,96.27(2), 90.00$ \\
\hline Unit cell volume $\left(\AA^{3}\right)$ & 1595.07 & 1636.94 & 2778.03 \\
\hline Density $_{\text {calc }}\left(\mathrm{g} \mathrm{cm}^{-3}\right)$ & 1.0694 & 1.2186 & 1.1513 \\
\hline Bilayer thickness, $l(\AA)$ & 28.86 & 29.33 & 28.80 \\
\hline Molecular area, $S\left(\AA^{2}\right)$ & 55.3 & 55.7 & 48.2 \\
\hline \multicolumn{4}{|l|}{ Hydrocarbon matrix: } \\
\hline chain packing mode & $\mathrm{O}^{\prime} \perp$ & $\mathrm{O} \|$ & HS (monoclinic) \\
\hline subcell: $a_{\mathrm{s}}, b_{\mathrm{s}}, c_{\mathrm{s}}(\AA)$ & $7.67(6), 5.12(3), 2.52(4)$ & $8.86(6), 8.98(6), 2.54(4)$ & $9.05(9), 8.58(1), 2.54(1)$ \\
\hline$\alpha_{s}, \beta_{s}, \gamma_{s}()$ & & $86(1), 92(1), 90(1)$ & $89.2(6), 101.6(1.4), 89.7(1)$ \\
\hline chain cross-section $\Sigma\left(\AA^{2}\right)$ & 19.5 & 19.4 & 19.2 \\
\hline chain tilt angle $\phi\left({ }^{\circ}\right)$ & 45 & 45 & 37 \\
\hline matrix thickness $(\AA)$ & 10.3 & 9.3 & 16.5 \\
\hline
\end{tabular}

*Anhydrous conditions.

\footnotetext{
1) In structural-conformational studies the sn-nomenclature is unrational and preferably replaced by the more general Fischer convention, which allows identical, comparable atom numbering in chiral antipodes, see Ref. [21].
} 


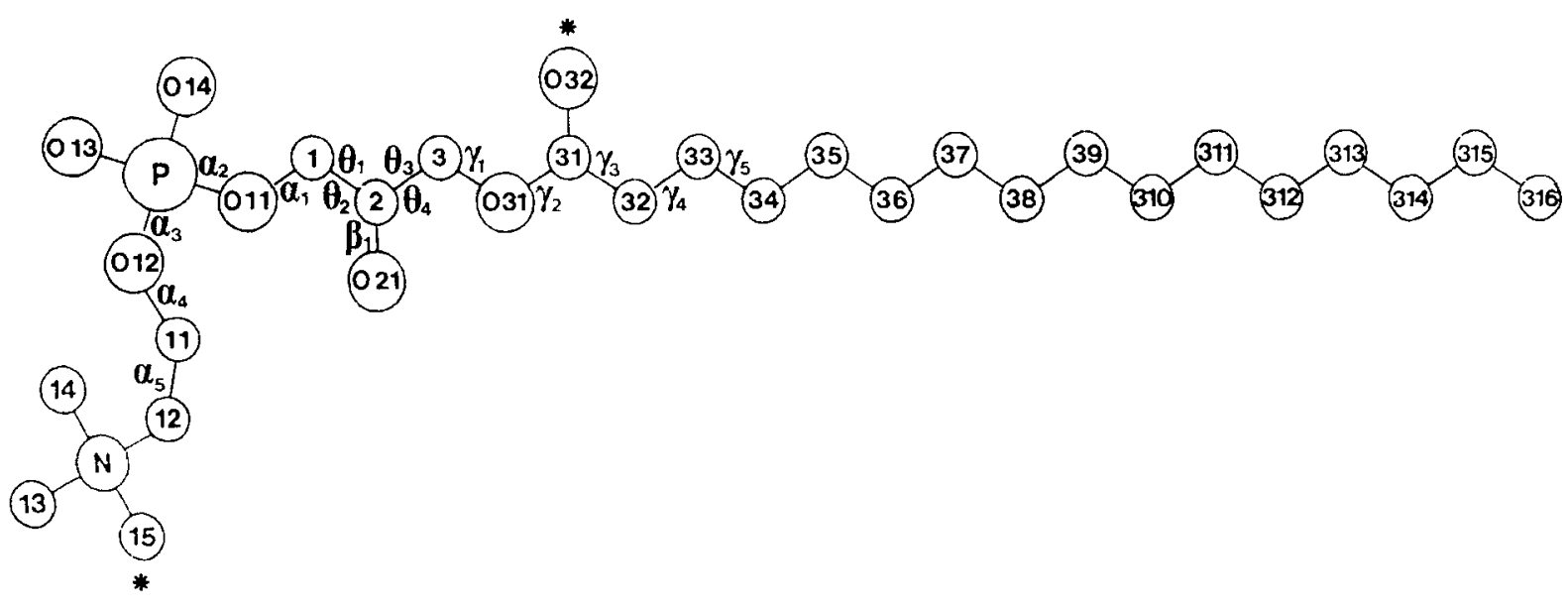

Fig. 1 Atom numbering and notations of torsion angles for palmitoyl-glycero-phosphocholine (PPC). In hexadecyl-glycero-phosphocholine (HPC) and palmitoyl-glycero-phospho- $N, N$-dimethylethanolamine $\left(\mathrm{PPEM}_{2}\right)$ the marked atoms $\mathrm{O} 32^{*}$ and $\mathrm{C} 15^{*}$, respectively, are replaced by hydrogens. For numbering conventions of glycerolipids in structural-conformational studies see Ref. [21]

but not of $\mathrm{PPEM}_{2}$, were found to accommodate one solvent molecule (water and chloroform, respectively) per lipid molecule. The crystals of PPC and HPC were unstable in moist air. They were handled in a dry atmosphere and mounted in sealed lithium glass tubes.

$\mathrm{X}$-ray reflections were recorded on an Enraf-Nonius CAD4F-11 diffractometer. The angular settings of 25 reflections were measured to calculate the lattice parameters. Intensity data for one hemisphere of reflection were collected by the $\omega / 2 \theta$ scan method using monochromatized $\mathrm{Cu} K x$-radiation. Three intensity control reflections were measured every $2 \mathrm{~h}$ to control crystal decay. The intensities were scaled to account for this decay $(10-30 \%)$. The numbers of independent reflections and observed reflections $(I>3 \sigma[I])$ for each compound are given in Table 1. All intensities were corrected for Lorentz and polarization effects but not for absorption or extinction.

The structures were solved by a combination of Patterson heavy atom method and direct methods using the program DIRDIF [17] which provided the non-hydrogen atom positions. All hydrogen atom positions except those of the water molecule in PPC were obtained from Fourier difference synthesis maps. Refinement was carried out by the full-matrix least-squares method using anisotropic temperature factors for the non-hydrogen atoms. The hydrogen atoms were assigned a common temperature factor $\left(B=5 \AA^{2}\right)$ but their atomic parameters were not refined. The structures were refined to $R$ - and $R_{w}$-values of about 0.10 and 0.15 , respectively (see Table 1). All calculations have been performed using mainly the XTAL 2.2 program system [18].

\section{Description of the structure}

Atomic coordinates and equivalent isotropic temperature factors $\left(U_{\mathrm{eq}}\right)$ of the non-hydrogen atoms have been deposited at the Cambridge Structure Database. Observed and calculated structure factors and anisotropic temperature factors can be obtained from this Department. The atom numbering and torsion angle notation, according to the convention of Sundaralingam [19], are shown in Fig. 1.

\section{Molecular packing}

The packing arrangements of the three lyso-PC compounds and the extension of the unit cells are shown in Fig. 2 in projections along the two short unit cell axes. In all three structures the molecules pack in a double-layer structure with interdigitating hydrocarbon chains and interdigitating head groups.

The unit cells of the enantiomeric PPC and HPC contain two different conformers, $\mathrm{A}$ and $\mathrm{B}$, which are orientated with their heads in opposite directions and pack separately in either half of the double-layer. As will be discussed below the phosphocholine groups of molecules A and B are related by a non-crystallographic centrosymmetry and are practically mirror images with respect to conformation and interaction pattern.

The unit cell of the racemic $\mathrm{PPEM}_{2}$ contains four molecules, which, however, are identical mirror image conformers related by crystallographic centro-symmetry. D- and L-enantiomers pack alternatingly within each 
Fig. 2 Molecular packing of lysophosphatidylcholines in views along the unit cell $a$-axis (left) and $b$-axis (right). The unit cells of the enantiomeric PPC and HPC lipids contain two conformers, $\mathrm{A}$ and $\mathrm{B}$, which are arranged in separate layers on either side of the double-layer structure. PPC accommodates a water molecule and $\mathrm{HPC}$ a chloroform molecule of solvation per lipid molecule. The unit cell of the racemic $\mathrm{PPEM}_{2}$ lipid contains four molecules which are related by centro-symmetry and arranged in pairs of $\mathrm{D} / \mathrm{L}$ mirror images on each side of the bilayer
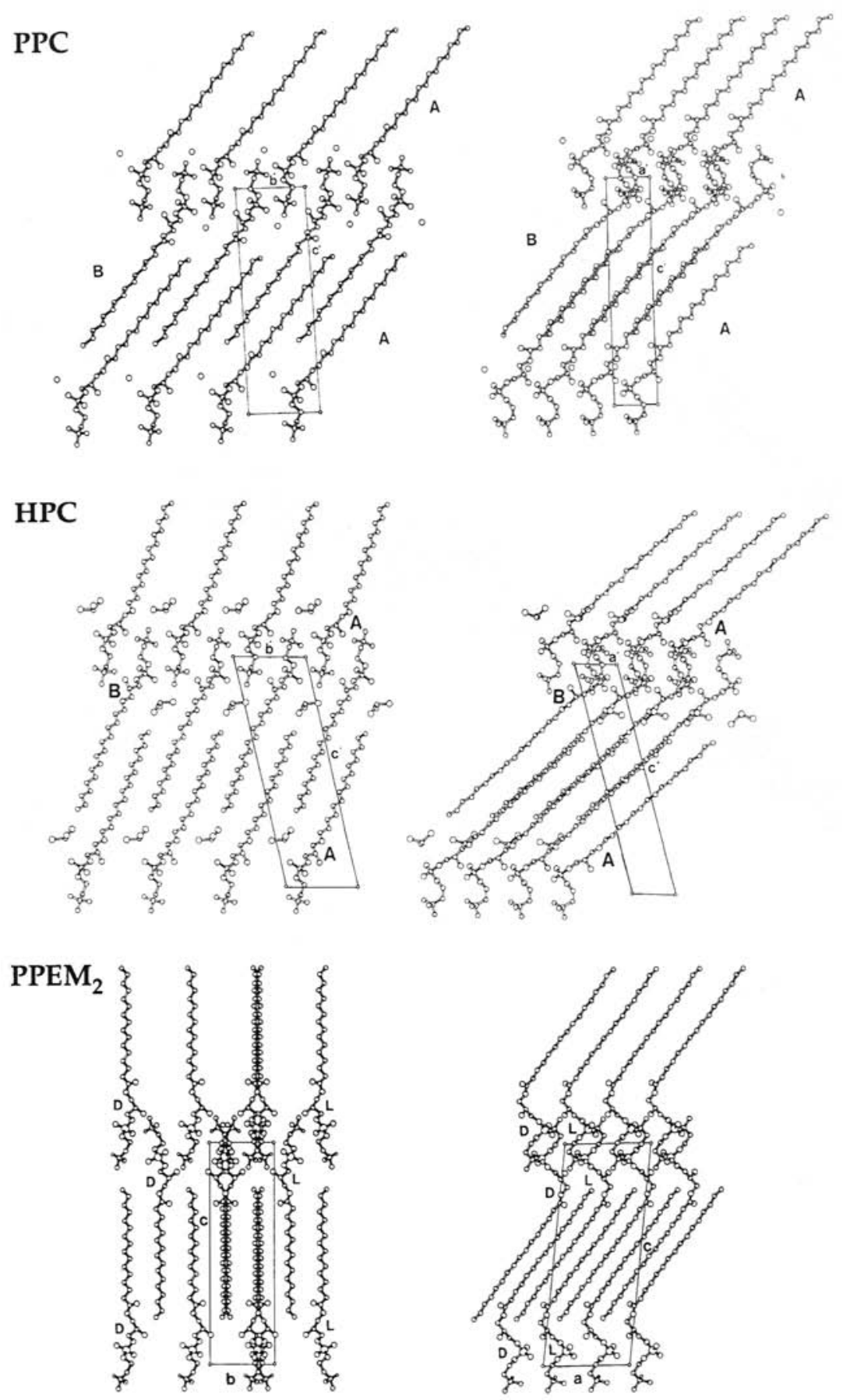

molecular layer and the unit cell contains two pairs of $\mathrm{D} / \mathrm{L}$-conformers, one pair at each side of the bilayer.

As shown in Fig. 2 the phosphoammonio head group dipoles extend perpendiculary to the layer plane and interdigitate with the head group dipoles of the adjacent lipid double-layer. This results in a single, about $7.5 \AA$ thick matrix of head group dipoles at the interface between two lipid double-layers. In these packing arrangements the molecular area in the layer plane ( $a b$-plane) is determined by the packing requirements of two antiparallel head group dipoles. For PPC and HPC the molecular packing cross-section $(S)$ is practically identical $\left(55.3\right.$ and $\left.55.7 \AA^{2}\right)$, while for $\mathrm{PPEM}_{2}$ it is distinctly smaller $\left(48.2 \AA^{2}\right)$. To this rather large molecular packing area the hydrocarbon 
chains accommodate by interdigitation with the chains of oppositely oriented molecules and, additionally, by a tilt $(\phi)$ with respect to the layer normal of $45^{\circ}$ in $\mathrm{PPC} / \mathrm{HPC}$ and $37^{\circ}$ in $\mathrm{PPEM}_{2}$. In PPC/HPC the $\mathrm{C}_{16}$-hydrocarbon chains thereby interdigitate by 13 carbon atoms only, leaving in extension of the chain ends a large cavity in which solvent molecules $\left(\mathrm{H}_{2} \mathrm{O}\right.$ and $\left.\mathrm{CHCl}_{3}\right)$ are accommodated. In $\mathrm{PPEM}_{2}$, on the other hand, the methyl chain ends interdigitate to the level of the glycerol carbon $\mathrm{C} 3$ of the opposite molecules, corresponding to a chain overlap of 18 atoms, not forming such an internal cavity. This partial or full interdigitation gives rise to a chain matrix layer which in PPC/HPC is only $10.3 / 9.3 \AA$ thick, while in $\mathrm{PPEM}_{2}$ it is $16.5 \AA$ thick.

In the three compounds the hydrocarbon chains are arranged according to different chain packing modes [20]: orthorhombic perpendicular $\left(\mathrm{O}^{\prime} \perp\right)$ in $\mathrm{PPC}$, orthorhombic parallel $(\mathrm{O} \|)$ in HPC and a hybrid packing mode $(H S)$ in $\mathrm{PPEM}_{2}$ (for subcell parameters see Table 1). The packing cross-sections of the chains $(\Sigma)$, perpendicular to their long axis are 19.5/19.4 $\AA^{2}$ in PPC/HPC and 19.2 $\AA^{2}$ in PPEM 2 . With reference to the rather thin chain matrix in PPC/HPC their hydrocarbon chains must be considered as well packed.

As already mentioned, the incomplete chain interdigitation in PPC/HPC creates a rather large cavity in exension of each hydrocarbon chain, which is $8.9 / 9.9 \AA$ in length and about $180 \AA^{3}$ in volume. In PPC this cavity is only partially filled up by a water molecule of solvation. The distance between the methyl chain end and the water molecule $(5.7 \AA)$ is considerably larger than a van der Waals contact. In an ordered chain matrix, however, the chains can slide past each other by integral zigzag units only. An interdigitation of the chains by another ethylene unit $\left(c_{s}=2.52 \AA\right)$ would bring the methyl chain end too close to the water molecule. This empty space in extention of each chain end causes a rather low specific density $\left(D_{\mathrm{c}}=1.069 \mathrm{~g} \mathrm{~cm}^{-3}\right)$ of the PPC crystal.

In HPC the cavity is, compared to PPC, one $\AA$ longer but more effectively filled by the much larger chloroform molecule. Judging from the high temperature factors of the chloroform atoms the fit of the solvate molecule is still not optimal. The heavy chlorine atoms, however, substantially increase the crystal density $\left(D_{\mathrm{c}}=1.219 \mathrm{~g} \mathrm{~cm}^{-3}\right)$ of HPC.

\section{Polar interactions}

The packing patterns and interactions of the phosphoammonio groups of the three lyso-lipids are shown in Fig. 3. As it becomes obvious from the view parallel to the double-layer interface (Figs. $2 \mathrm{~A}$ and $3 \mathrm{~A}$ ) the $\mathrm{P}-\mathrm{N}$ dipoles extend almost perpendiculary to the layer plane and interdigitate with those of the next double layer. Thereby the $(+)$ ammonio groups of one layer protrude to the level of the $(-)$ phosphate group of the adjacent head group layer. In this layer of interdigitating head groups two layers of $(+)$ and $(-)$ charges are formed, $4.2 \AA$ apart.

In PPC/HPC the pseudo-centrosymmetry of the head groups entails that the packing and interaction pattern of one layer of phosphate (A) and choline (B) groups is a mirror image of the corresponding phosphate $(B)$ and choline (A) pattern of the other half of the interdigitating headgroup layer. Note, however, that this mirror symmetry is not perfect and minor differences in the length of intermolecular contacts exists for molecules $A$ and $B$.

In $\mathrm{PPEM}_{2}$ the head groups display a rather similar interdigitating packing arrangement, however, the D- and L-enantiomers are true configurational/conformational mirror images and have identical centro-symmetry related packing contacts and interaction patterns.

Interestingly, in all three lysolipids short electrostatic contacts are established only between distinct pairs of the $(+)$ ammonio and (-) phospho groups, rather than towards several surrounding neighbor molecules. The ammonio nitrogen in PPC/HPC thus makes two short lateral contacts $(<4.0 \AA)$ towards $\mathrm{O} 12$ and $\mathrm{O} 13$ of a neighboring phosphate group and a third short contact $(3.7 \AA)$ to the glycerol $\mathrm{O} 21$ of another neighbor molecule.

What is most significant for all phospholipids, however, is that the unesterified phosphate oxygens $\mathrm{O} 13$ and O14 strongly attract hydrogen donor groups. In all three lyso-compounds one hydrogen donor bond is provided by the fri glycerol hydroxyl group $\mathrm{O} 21 \rightarrow \mathrm{O} 14(\approx 2.7 \AA)$, not intramolecularly, but intermolecularly from an a-translated neighbor molecule. The other hydrogen bond towards O13, however, comes in the three lipids from different donors. In PPC the water molecule of hydration (W1) directs one hydrogen bond $(2.9 \AA)$ to O13 (but does not engage its second hydrogen). Note, that this water molecule was retained or attracted, although PPC was dried over $\mathrm{P}_{2} \mathrm{O}_{5}$ and crystallized under anhydrous conditions.

Surprisingly, in HPC the polarized $\mathrm{C}-\mathrm{H}$ bond of chloroform acts as a hydrogen donor $(\approx 3.1 \AA)$ towards O13. Finally in $\mathrm{PPEM}_{2}$ the not methyl-substituted ammonio hydrogen interacts with $\mathrm{O} 13$ of a neighboring interdigitating phosphate group. This $\mathrm{N}-\mathrm{H} \rightarrow \mathrm{O} 13$ hydrogen bond $(2.7 \AA)$ together with an $\mathrm{N}(+) \rightarrow \mathrm{O} 11$ ionic bond (3.4 $\AA$ ) directed from the ammonio group towards one neighboring phosphate group results for $\mathrm{PPEM}_{2}$ in a closer contact between head group pairs and a substantial reduction $(13 \%)$ of the molecular packing crosssection compared to PPC/HPC (see Table 1). 
Fig. 3 Packing and interactions of the zwitter-ionic head group dipoles of PPC, $\mathrm{HPC}$ and $\mathrm{PPEM}_{2}$ in views parallel (left) and perpendicular (right) to the bilayer interface. Hydrogen bonds are indicated by dotted lines, electrostatic and polar contacts by broken lines. Contact distances are given in $\AA$
PPC
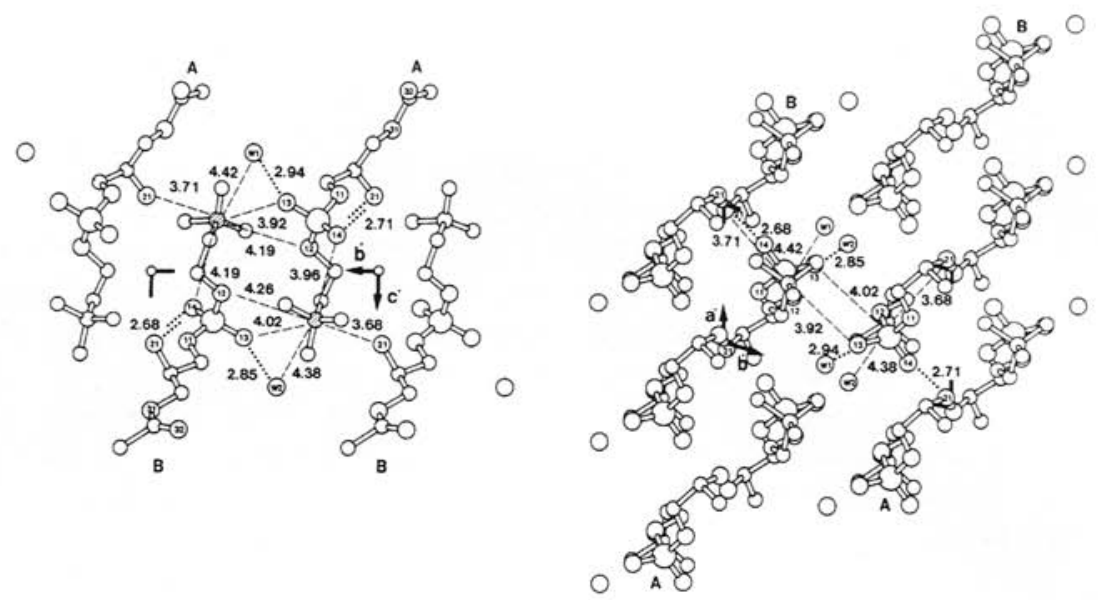

HPC
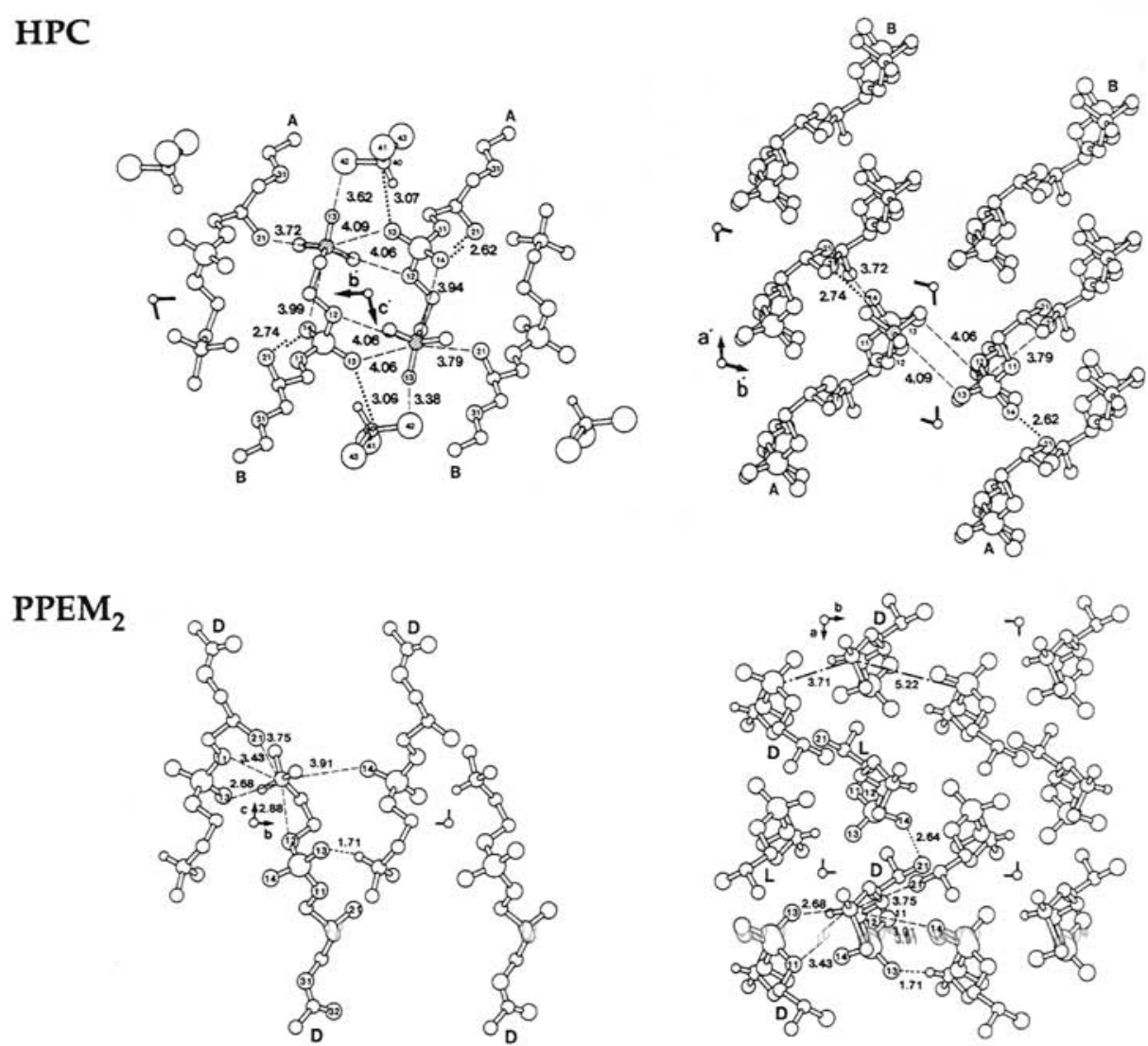



\section{Molecular conformation}

The conformations of molecules A and B of PPC/HPC and of the D- and L-enantiomer of $\mathrm{PPEM}_{2}$ are shown in Fig. 4. Torsion angles of the molecules in comparison to corresponding diacyl derivatives are given in Table 2. For notations of the torsion angles see Fig. 1 and Refs. [19, 21]. The terminology of Klyne and Prelog [22] is used to describe conformational ranges.
PPC/HPC: Although the PPC/HPC-molecules have the natural $D$-configuration the polar part of molecules $\mathrm{A}$ and $\mathrm{B}$ adopts a quasi-symmetric mirror image conformation up to glycerol carbon C2. The two glycerol oxygens $\mathrm{O} 11$ and $\mathrm{O} 21$ of molecules $\mathrm{A}$ and $\mathrm{B}$ still retain mirror image geometry $\left(\theta_{2}=+\mathrm{sc}(\mathrm{A})\right.$ and $\left.-\mathrm{sc}(\mathrm{B})\right)$, since $\mathrm{O} 21$ is involved in the pseudo-centrosymmetric interaction pattern of the head group dipoles. Due to chirality the centro-symmetry is broken at glycerol carbon $\mathrm{C} 2$ and the 
glycerol carbons $\mathrm{C} 3$ in molecules $\mathrm{A}$ and $\mathrm{B}$ are oriented differently $\left(\theta_{1}=-\mathrm{sc}\right.$ and $\left.a p\right)$ with respect to the polar group. On the other hand, the glycerol-attached hydrocarbon chains have from $\mathrm{C} 3$ onwards a rather identical antiplanar conformation but different orientations of their chain planes with respect to their head groups. In PPC A the orientation of the chain plane is additionally effected
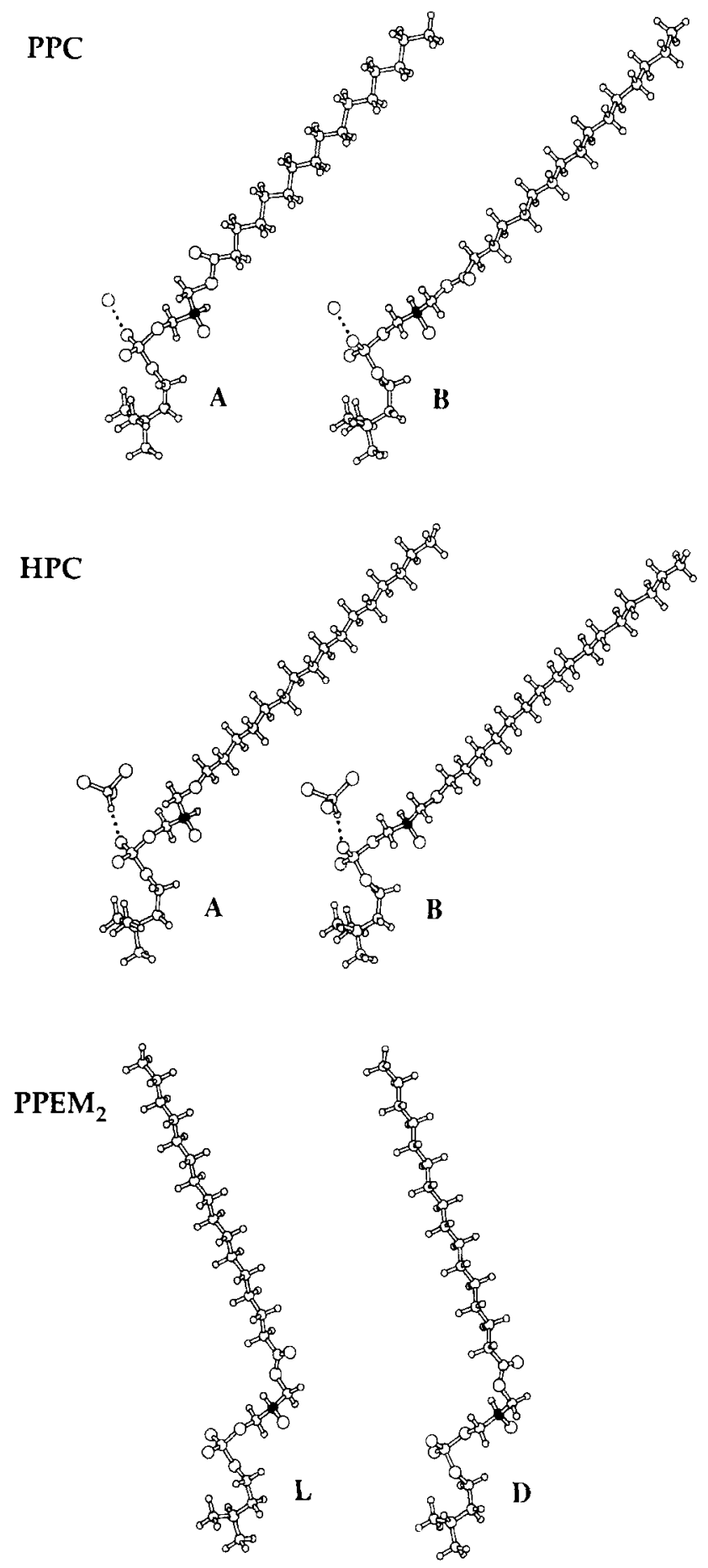

by torsion $\gamma_{1}=\mathrm{ac}$, to allow an accommodation of the chains in the $\mathrm{O}^{\prime} \perp$ chain matrix.

PPEM: As evident from Fig. 4 and torsion angles in Table 2 the D- and L-enantiomers of $\mathrm{PPEM}_{2}$ are true mirror images and resemble with respect to their polar group conformation the molecules $\mathrm{B}$ and $\mathrm{A}$ of PPC/HPC. The conformational similarity of the $\mathrm{PPEM}_{2}$ L-enantiomer with the D-enantiomeric PPC/HPC molecule A comprises the $\alpha$-chain only, while for the $\mathrm{PPEM}_{2} \mathrm{D}$ enantiomer and the D-enantiomeric PPC/HPC molecule $\mathrm{B}$ it also includes the glycerol torsions $\theta_{1} / \theta_{2}$. In $\mathrm{PPEM}_{2}$, a significant difference exists in the torsions $\theta_{3} / \theta_{4}=$ - sc/ap (D) and sc/ap (L), which turn the glycerol oxygen $\mathrm{O} 3$ and the rest of the $\gamma$-chain perpendicularly to the direction of the glycerol chain. Compared to PPC/HPC this bend at the $\mathrm{C} 2-\mathrm{C} 3$ glycerol bond reverses the direction of the chain tilt in $\mathrm{PPEM}_{2}$.

\section{Discussion}

As mentioned earlier, in the crystal stuctures of phosphatidylethanolamines and phosphatidylcholines solved so far the head groups exhibit similar conformations but very different packing patterns [21]. Among the PC-compounds only 3-lauroyl-2-deoxy-glycero-1-phosphocholine [13] shows the "expected" layer-parallel orientation of the $\mathrm{P}-\mathrm{N}$ dipoles, while in 2,3-dimyristoyl- $D L$-glycero-1-phosphocholine [15] the head group layer is folded into a space-saving saw-tooth arrangement with a packing area of $38 \AA^{2}$ per molecule. In 3-octadecyl-2-methyl- $D L$-glycero-1-phosphocholine [14], on the other hand, the $\mathrm{P}-\mathrm{N}$ dipoles are aligned layer-parallelly, but interdigitate with those of the adjacent bilayer. This results in a very large packing cross-section per molecule $\left(74 \AA^{2}\right)$ corresponding to a packing area of almost two phosphocholine groups.

The presented structures of the three lyso-PCs show still another head group arrangement with extended,

Fig. 4 Molecular conformation of conformer A (left) and conformer $B$ (right) of PPC and HPC together with their hydrogen bonded molecules of solvation (water and chloroform, respectively), and of the two mirror image enantiomers $D$ and $L$ of PPEM ${ }_{2}$. The molecules are shown with their polar heads in an identical projection. perpendicular to their (pseudo)-mirror plane, in order to demonstrate the mirror image conformation of the headgroups including the glycerol atoms $\mathrm{C} 1-\mathrm{C} 2 \mathrm{O} 21$. For PPC and $\mathrm{HPC}$ the pseudocentro-symmetry is broken at the chiral glycerol carbon $\mathrm{C} 2$ (in black). The hydrophobic part of the molecules beyond $\mathrm{C} 2$ adopts an anti-planar conformation, however, the orientation of the chain planes with respect to the polar part differs in the different conformers. The mirror image conformers D and $\mathrm{L}$ of PPEM $\mathrm{P}_{2}$ have head group conformations identical with molecules B and A of PPC/HPC, but different torsions $\left(\theta_{3} / \theta_{4}\right)$ about the glycerol $\mathrm{C} 2$ C3 bond, producing a reverse tilt of the hydrocarbon chains 
Table 2 Torsion angles of PPC, HPC and $\mathrm{PPEM}_{2}$. For comparison the torsion angles of the corresponding double-chain lipids DMPC [15] and DLPEM ${ }_{2}{ }^{\mathrm{b}}$ [23] are given (in italics). The letters A or B after the abbreviated compound name refer to the two conformationally unique molecules in the unit cells of PPC and HPC and the letters D and L to the configurational/conformational mirror image molecules in the racemic $\mathrm{PPEM}_{2}$ structure. For notation of torsion angles see Fig. 1

\begin{tabular}{|c|c|c|c|c|c|c|c|c|c|c|c|c|c|c|c|c|c|}
\hline & $\alpha_{1}$ & $\alpha_{2}$ & $\alpha_{3}$ & $\alpha_{4}$ & $\alpha_{5}$ & $\theta_{1}$ & $\theta_{2}$ & $\theta_{3}$ & $\theta_{4}$ & $\beta_{1}$ & $\beta_{2}$ & $\beta_{3}$ & $\beta_{4}$ & $\gamma_{1}$ & $\gamma_{2}$ & $\gamma_{3}$ & $\gamma_{4}$ \\
\hline PC & 156 & 53 & 70 & 144 & -77 & -58 & 62 & -167 & 73 & & & & & 166 & 169 & 170 & -179 \\
\hline $\mathrm{HP}$ & 161 & 61 & 64 & 138 & -69 & -68 & 58 & -170 & 61 & & & & & 3 & 174 & 178 & 179 \\
\hline$D M P C A$ & 163 & 6 & 6 & 143 & -64 & 5 & 177 & -178 & 63 & 82 & 172 & 81 & 45 & -177 & 168 & -173 & 178 \\
\hline PPC B & 168 & -76 & $\begin{array}{l}-50 \\
-59\end{array}$ & -149 & 72 & 179 & -63 & -166 & 77 & & & & & -169 & -170 & 175 & -174 \\
\hline HPC B & 167 & -69 & -56 & -141 & 69 & 169 & -73 & -170 & 70 & & & & & 176 & -179 & -179 & -179 \\
\hline$D M P C B$ & 177 & -74 & -47 & -150 & 54 & 168 & -80 & 166 & 51 & 120 & 179 & -134 & 67 & 102 & 176 & 180 & 180 \\
\hline $\mathrm{PPEM}_{2} \mathrm{D}$ & 174 & -61 & -64 & -163 & 55 & 166 & -62 & -68 & 162 & & & & & -113 & 179 & -153 & -178 \\
\hline $\mathrm{PPEM}_{2} \mathrm{~L}$ & -174 & 61 & 64 & 163 & -55 & -166 & 62 & 68 & -162 & & & & & 113 & -179 & 153 & 178 \\
\hline$D L P E M_{2}$ & 179 & 65 & 54 & 144 & -96 & 176 & -66 & 56 & -60 & 148 & 173 & -57 & 176 & 129 & -167 & 166 & 175 \\
\hline
\end{tabular}

${ }^{\text {a) }} \mathrm{DMPC}=$ 2,3-dimyristoyl- $D$-glycero-1-phosphocholine dihydrate.

${ }^{b)} \mathrm{DLPEM}_{2}=2,3$-dilauroyl- $D L$-glycero-1-phospho- $N, N$-dimethyletanolamine.

Fig. 5 Comparison of the molecular packing of PPEM$_{2}$ and the corresponding doublechain lipid dilauroyl- $D L-$ glycero-phospho- $N, N$ dimethylethanolamine [23]. The monoacyl- and diacyl-lipid show practically identical packing and interaction pattern, but the hydrocarbon chains of the double-chain lipid do not interdigitate

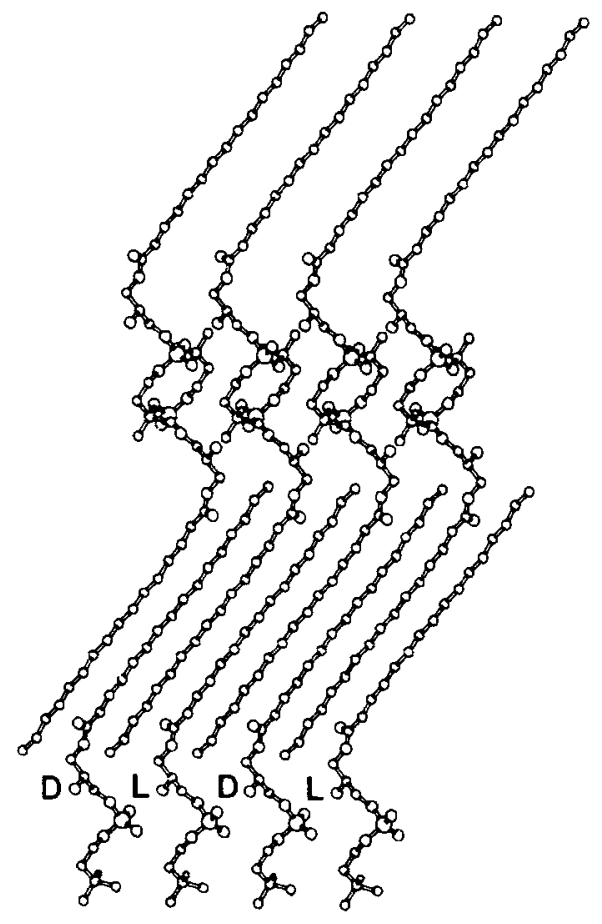

$\mathrm{PPEM}_{2}$
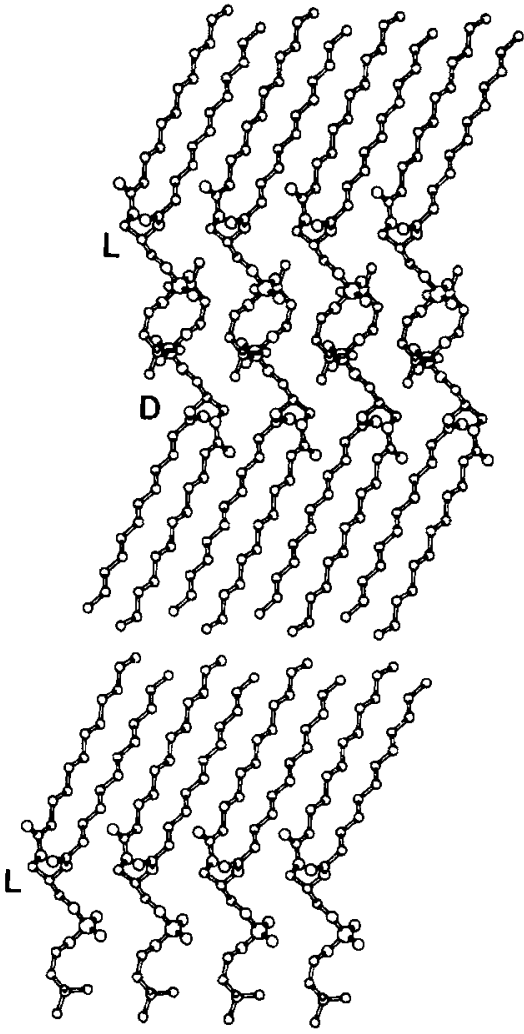

DLPEM $_{2}$ layer-perpendicular $\mathrm{P}-\mathrm{N}$ dipoles which interdigitate with those of the adjacent bilayer. This type of interdigitating head group dipoles, which can only arise in stacked multilamellar structures, is not specific for the reported lysolipids. The same packing arrangement with interdigitating head group dipoles has also been found in the crystal structures of 2,3-dilauroyl- $D L$-glycero-1-phospho- $N, N$-dimethyl-ethanolamine $\left(\right.$ DLPEM$\left._{2}\right)$ [23] and 2,3-dilauroyl$D L$-glycero-1-phospho- $N$-monomethylethanolamine $\left(\right.$ DLPEM $\left._{1}\right)($ Pascher and Sundell, unpublished). Interest- 
ingly, the double-chain lipid DLPEM $\mathrm{D}_{2}$ exhibits the same packing arrangement as $\mathrm{PPEM}_{2}$, with the exception that the chain matrix of DLPEM 2 does not interdigitate (Fig 5).

It appears that in multilamellar structures the interdigitation of $\mathbf{P}-\mathbf{N}$ dipoles is energetically favorable, as it gives rise to more effective lateral head group interaction at the bilayer interface. In structures with layer-parallel head groups the corresponding interactions become distributed to two separate head group layers. The two types of head group arrangements, however, do not significantly effect the molecular packing cross-section. In PPC/HPC molecular area $\left(55 \AA^{2}\right)$ is rather similar to that of 3-lauroyl2-deoxy-glycero-1-phosphocholine $\left(52 \AA^{2}\right)$ with layer-parallel dipoles, as in the interdigitating phosphate/ choline pattern the choline groups of one lipid layer are replaced by those protruding from the adjacent lipid layer.
An interesting feature that emerges from these crystal structure analyses is the fact that, despite the great variety of observed arrangements of head group dipoles, the zwitterionic phosphoethanolamine/choline head groups always adopt a typical preferred conformation, that usually occurs in coexisting, energetically equivalent mirror image conformers. This characteristic conformation, which according to $a b$ initio calculations $[24,25]$ is favored by intrinsic energetics is not effected by hydration, and, as confirmed by this study, not by the degree of $N$-methylation or the type and attachment of the hydrocarbon chains.

Acknowledgments This work is supported by the Swedish Medical Research Council (grant 0006), the Alice and Knut Wallenberg Foundation and the Skandinaviska Enskilda Banken Foundation.

\section{References}

1. White DA (1973) In: Ansell GB, Hawthorn JN, Dawson RMC (eds) Form and Function of Phospholipids. Elsevier, Amsterdam

2. Weltzien HU, Munder PG (1983) In: Mangold HK, Paltauf $F$ (eds) Etherlipids. Academic Press, New York

3 Eibl H (1984) Angew Chem Int Ed 23:257-271

4. Pinckard RN, McManus LM, Hanahan DJ (1982) In: Wiessman G (ed) Advances in Inflammation Research. Vol 4. Raven Press. New York, pp $147 \cdot 180$

5. Shukla SD, Hanahan DJ (1984) Arch Biochem Biophys 232:458 466

6. Berger MR. Muschiol C. Schmäht D. Unger C. Eibl H (1985) In: Unger C. Eibl $\mathrm{H}$, Nagel $\mathrm{G}$ (eds) Die Zellmembran als Angriffspunkt in der Tumortherapic. Aktuelle Onkologie 34. Zuckschwert Verlag, München-Bern-Wien, pp $27-36$

7. Eibl H. Kaufmann-Kolle P (1995) J Liposome Res 5:131 148

8. Arvidson C, Brentel I, Kahn A. Lindblom G, Fontell K (1985) Eur J Biochem 152:753-759
9. Larsson K (1994) In: Larsson K (ed) Lipids - Molecular Organization, Physical Functions and Technical Applications, Chs $2 \& 3$. The Oily Press, Dundee

10. Mattai J, Shipley GG (1986) Biochim Biophys Acta 859:257 265

11. Jain MK, Crecely RW, Hille JDR, de Haas GH. Gruner SM (1985) Biochim Biophys Acta 813:68-76

12. Abrahamsson S. Pascher I (1966) Acta Crystallogr 21:79-87

13. Hauser H, Pascher I, Sundell S (1980) J Mol Biol 137:249-264

14. I. Pascher I, Sundell S, Eibl H, Harlos K (1986) Chem Phys Lipids 39:53-64

15. Pearson RH, Pascher I (1979) Nature (London) 281:499-501

16. Eibl H. Woolley P (1988) Chem Phys Lipids 47:63-68

17. Beurskens PT, Bosman WPJH, Gould RO, Van den Hark TEM, Prick PAJ (1978) Technical Report 1978/1, Crystallography Laboratory; Toernvooiveld. 6525 ED Nijmegen, The Netherlands
18. Hall SR, Stewart JM (eds) (1988) XTAL2.2 User's Manual, Universities of Western Australia and Maryland

19. Sundaralingam M (1972) Ann NY Acad Sci USA 195:324-355

20. Abrahamsson S, Dahlén B, Löfgren $H$, Pascher I (1978) Prog Chem Fats other Lipids 16:125-143

21. Pascher I, Lundmark M, Nyholm P-G, Sundell S (1992) Biochim Biophys Acta 1113:339--373

22. Klyne W, Prelog V (1960) Experientia 16:512-523

23. Pascher I, Sundell S (1986) Biochim Biophys Acta 855:68-78

24. Landin J, Pascher I, Cremer D (1995) J Phys Chem 99:4471-4485

25. Landin J, Pascher I, Cremer D (1997) J Phys Chem 101:2996-3004 